\title{
Practical PET Respiratory Motion Correction in Clinical PET/MR
}

\author{
Richard Manber ${ }^{1}$, Kris Thielemans ${ }^{2}$, Brian F. Hutton ${ }^{2,3}$, Anna Barnes ${ }^{2}$, Sébastien Ourselin ${ }^{4}$, Simon Arridge ${ }^{4}$, \\ Celia O'Meara ${ }^{5}$, Simon Wan², and David Atkinson ${ }^{1}$ \\ ${ }^{1}$ Division of Medicine, Centre for Medical Imaging, University College London, London, United Kingdom; ${ }^{2}$ Institute of Nuclear \\ Medicine, UCL and UCL Hospitals, London, United Kingdom; ${ }^{3}$ Centre for Medical Radiation Physics, University of Wollongong, \\ New South Wales, Australia; ${ }^{4}$ Centre for Medical Imaging Computing, Faculty of Engineering, University College London, London, \\ United Kingdom; and ${ }^{5}$ Sidra Medical and Research Center, Doha, Qatar
}

Respiratory motion during PET acquisition may lead to blurring in resulting images and underestimation of uptake parameters. The advent of integrated PET/MR scanners allows us to exploit the integration of modalities, using high spatial resolution and highcontrast MR images to monitor and correct PET images degraded by motion. We proposed a practical, anatomy-independent MRbased correction strategy for PET data affected by respiratory motion and showed that it can improve image quality both for PET acquired simultaneously to the motion-capturing MR and for PET acquired up to $1 \mathrm{~h}$ earlier during a clinical scan. Methods: To estimate the respiratory motion, our method needs only an extra 1-min dynamic MR scan, acquired at the end of the clinical PET/MR protocol. A respiratory signal was extracted directly from the PET listmode data. This signal was used to gate the PET data and to construct a motion model built from the dynamic MR data. The estimated motion was then incorporated into the PET image reconstruction to obtain a single motion-corrected PET image. We evaluated our method in 2 steps. The PET-derived respiratory signal was compared with an MR measure of diaphragmatic displacement via a pencilbeam navigator. The motion-corrected images were compared with uncorrected images with visual inspection, line profiles, and standardized uptake value (SUV) in focally avid lesions. Results: We showed a strong correlation between the PET-derived and MRderived respiratory signals for 9 patients, with a mean correlation of 0.89 . We then showed 4 clinical case study examples $\left({ }^{18} \mathrm{~F}-\mathrm{FDG}\right.$ and ${ }^{68} \mathrm{Ga}$-DOTATATE) using the motion-correction technique, demonstrating improvements in image sharpness and reduction of respiratory artifacts in scans containing pancreatic, liver, and lung lesions as well as cardiac scans. The mean increase in peak SUV $\left(S_{\text {peak }}\right)$ and maximum SUV $\left(S_{\text {SUax }}\right)$ in a patient with 4 pancreatic lesions was $23.1 \%$ and $34.5 \%$ in PET acquired simultaneously with motion-capturing MR, and $17.6 \%$ and $24.7 \%$ in PET acquired $50 \mathrm{~min}$ before as part of the clinical scan. Conclusion: We showed that a respiratory signal can be obtained from raw PET data and that the clinical PET image quality can be improved using only a short additional PET/MR acquisition. Our method does not need external respiratory hardware or modification of the normal clinical MR sequences.

Received Jan. 13, 2015; revision accepted Apr. 2, 2015.

For correspondence or reprints contact: Richard Manber, Institute of Nuclear Medicine, 5th Floor, Tower, University College Hospital, 235 Euston Rd., London, NW1 2BU, U.K.

E-mail: r.manber@ucl.ac.uk

Published online May 7, 2015.

COPYRIGHT (c) 2015 by the Society of Nuclear Medicine and Molecular Imaging, Inc.
Key Words: motion correction; PET/MR; lesion detection; lesion quantification

J Nucl Med 2015; 56:890-896

DOI: 10.2967/jnumed.114.151779

\section{$\mathbf{R}$} ring in resulting images and errors in quantification $(1,2)$. Areas in the upper abdomen and thorax are particularly adversely affected. In the case of pathologic lesions, PET images may help characterize the etiology based on standardized uptake value (SUV), provide information for monitoring of response to therapy, and help to delineate lesion boundary and calculation of lesion volumes for radiotherapy planning (3), all of which can be adversely affected by respiratory motion.

Motion correction can be achieved by gating (splitting data into respiratory states) $(2,4)$, reconstructing separate images, and registering to a common respiratory state (5). A review of this method is given by Nehmeh and Erdi (1). This motion-correction technique requires good contrast to noise in each gated image for accurate registration results. Obtaining good contrast to noise becomes difficult because there is competing interest to reduce scan time for increased patient throughput and to reduce tracer activity injected for reduced patient dose, leading to low count statistics and lower contrast to noise in each gate.

The recent advent of PET/MR scanners allows us to exploit the simultaneity of the modalities using high spatial resolution and high-contrast MR images to track respiratory motion and correct PET data, without additional radiation exposure. Tagged MR is one such technique, which uses a special pulse sequence to create temporary features (tags) in tissue, which deform and can be tracked in images as the anatomy deforms $(6,7)$. An alternative technique is to acquire quick motion-capturing multislice 2-dimensional (2D) MR images of the thorax and calculate deformation fields by registration of the images (8). A similar method has also been proposed using 3-dimensional dynamic MR, in which a patient-specific MR motion model is built to capture breathing patterns linked to a $2 \mathrm{D}$ image navigator, with the method tested on simulated PET/MR data (9).

Although these methods show an improvement in PET image quality, all require a change to the otherwise intended PET/MR protocol to be able to collect the respiratory signal or MR-derived motion model in a clinical setting. Measuring the respiratory signal using external monitoring devices, such as the Real-Time Position Management (Varian Medical Systems Inc.), spirometer, 
TABLE 1

Overview of 4 Patients with Varying ROls and Administered Tracer

\begin{tabular}{|c|c|c|c|c|}
\hline Patient no. & Sex & Age (y) & ROls & Tracer \\
\hline 1 & M & 41 & $\begin{array}{c}5 \text { liver lesions, } \\
1 \text { lung lesion }\end{array}$ & ${ }^{68} \mathrm{Ga}$ \\
\hline 2 & $\mathrm{~F}$ & 48 & 4 pancreatic lesions & ${ }^{68} \mathrm{Ga}$ \\
\hline 3 & $M$ & 69 & Cardiac & ${ }^{18} \mathrm{~F}$ \\
\hline 4 & M & 66 & Lung-liver edge artifact & ${ }^{68} \mathrm{Ga}$ \\
\hline Mean $\pm S D$ & & $56 \pm 14$ & & \\
\hline
\end{tabular}

or pressure belt, requires time for set-up and readjustment and can fail due to mispositioning, patient movement, or poor calibration. Another respiratory measure, the gold-standard MR pencil-beam navigator, needs to either run continuously or be inserted into the MR sequence being run in parallel with the PET, but this needs to be set up in advance of the scan, can create artifacts in MR images near the diaphragm, and may increase scan time. Furthermore, any bespoke motion-capturing MR sequence has to be acquired separately from any clinical diagnostic MR and thus may increase overall scan time further.

We proposed a respiratory motion-correction method that required no external hardware to provide a respiratory signal and no change to the imaging examination except for the addition of a short PET/MR sequence after the clinical acquisition. We proposed that sufficient data for respiratory correction can be acquired in just $1 \mathrm{~min}$. The acquisition was used to build a patient-specific respiratory motion model and then used to motion-correct the clinical PET data of any duration. Our method used only standard MR sequences and image registration techniques. The approach is practical and anatomically general, applicable to any type of thorax/abdomenrelated motion caused by respiration (e.g., lung/liver/pancreatic lesions and cardiac data).

In this work, we first performed a validation of a PET-derived respiratory signal, by comparison with an absolute measure of diaphragmatic displacement using an MR pencil-beam navigator, on 9 subjects in vivo. Next, we used this PET-derived signal to drive a motion model formed by nonrigid registration of MR slices

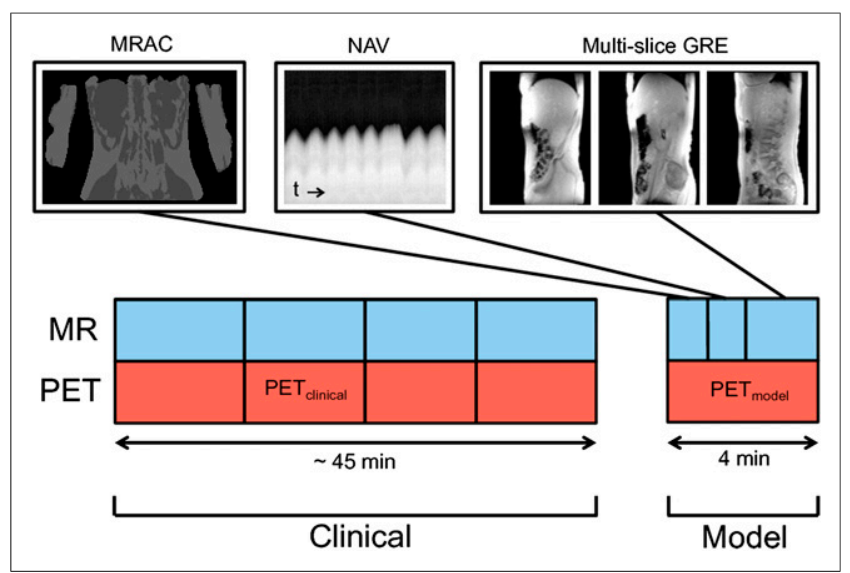

FIGURE 1. Motion model PET/MR sequence acquired after clinical scan: MRAC, pencil-beam navigator (NAV), and multislice gradient echo (GRE) sequence. acquired rapidly after the main scan. This motion was then incorporated into the PET reconstruction.

To evaluate our method, motion-corrected images were compared with uncorrected images with line profiles and changes in standardized uptake value (SUV) through regions of high tracer uptake for selected in vivo case study examples.

\section{MATERIALS AND METHODS}

\section{Data}

All data were acquired using an integrated 3-T PET/MR system (Biograph mMR; Siemens Healthcare), at University College London Hospital, U.K. Additional data were acquired as part of calibration and service development protocols. Patients consented to the use of their data for research purposes. PET data processing (unlisting, reconstruction, etc.) was performed with STIR (Software for Tomographic Image Reconstruction) (10). All other analysis was performed with Matlab (The MathWorks, Inc.), and Medical Image Registration Toolbox (11) was used in Matlab for registration.

\section{Respiratory Signal Validation}

For the validation component of the study, we compared our PETderived respiratory signal with an absolute measure of diaphragmatic displacement using an MR pencil-beam navigator, on 9 subjects in vivo. The respiratory signal is extracted from PET data as described below.

\section{PET-Derived Signal Extraction}

It has been shown that a respiratory signal can be extracted from raw PET list-mode data with principal component analysis (PCA) (12). If areas of sufficient contrast in the tracer uptake in a PET scan are moving, this movement will be detectable in the raw PET data.

First, the PET list-mode file is unlisted into $N$ short (0.4-s duration) low-spatial-resolution sinogram frames to form a 4-dimensional sinogram series. Sinograms are then spatially smoothed, a scale factor is applied to account for tracer kinetics, the Freeman-Tukey transformation is applied to approximately convert Poisson noise to gaussian, and then finally PCA is executed. With PCA, each sinogram in the series is approximated as:

$$
s_{i} \approx \bar{s}+\sum_{k=1}^{K} w_{i, k} p_{k}
$$

where $s_{i}$ is sinogram $i \in 1 \ldots N, \bar{s}$ is the mean of all sinograms, $p_{k}$ is principal component $(\mathrm{PC}) \in 1 \ldots K$, and $w_{i, k}$ is the scalar weight factor for sinogram $i$, PC $k$. For $1 \mathrm{PC}$, each sinogram in the time series therefore has a single weight factor, calculated as the voxelwise multiplication of the PC with the difference between the sinogram and sinogram mean,

$$
w_{i, k}=p_{k} \cdot\left(s_{i}-\bar{s}\right)
$$

These weights $w_{i, 1}$ for PC $k=1$ are then temporally smoothed, and these provide the respiratory signal.

\section{Study Design: Respiratory Signal Validation}

Data were collected on 9 patients (age range, 34-80 y; mean age $\pm \mathrm{SD}, 60 \pm 15 \mathrm{y}$ ) immediately after the clinical PET/MR scan. These patients had a range of diseases and were imaged with either ${ }^{18} \mathrm{~F}-\mathrm{FDG}(n=6)$ or ${ }^{68} \mathrm{Ga}$-DOTATATE $(n=3)$. The protocol consisted of PET list-mode with an MR pencil-beam navigator (continuously acquired; scout mode; repetition time, $150 \mathrm{~ms}$ ), placed on the right hemidiaphragm at the lung-liver edge. The PET and MR data were acquired concurrently (overlap time range, 93-180 s; mean overlap time \pm SD, $144 \pm 22 \mathrm{~s})$. 


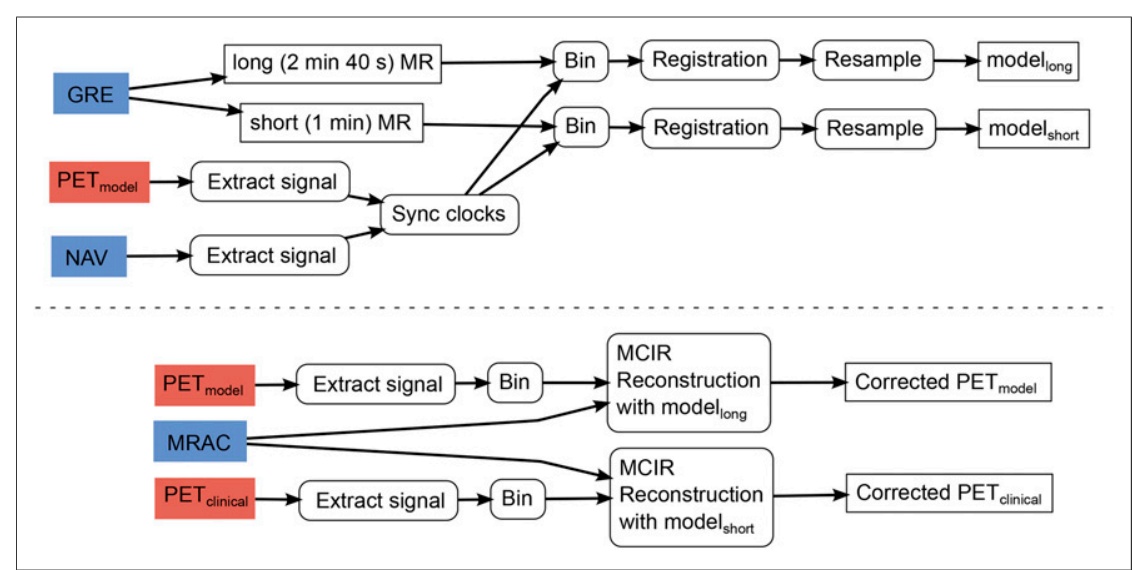

FIGURE 2. Overview of data workflow: building motion models, then using them in PET reconstructions. NAV = pencil-beam navigator.

\section{Analysis: Respiratory Signal Validation}

The PET signal was extracted from the list-mode data according to Equations 1 and 2 from each PET acquisition. An MR-derived signal was extracted by applying an edge-detection algorithm to the pencilbeam navigator image to find the relative height of the lung-liver edge at a temporal resolution of $150 \mathrm{~ms}$. Correlation between the 2 signals was calculated with the 1-dimensional Pearson correlation coefficient. First, the 2 signals were interpolated to a temporal resolution of $0.1 \mathrm{~s}$, and then correlation $\rho_{p, m}$ was found with

$$
\rho_{p, m}=\frac{\operatorname{COV}(p, m)}{\sigma_{p} \sigma_{m}}
$$

where $\sigma_{p}$ and $\sigma_{m}$ are the SD of the PET- and MR-derived signals, respectively, and COV is the covariance of the 2 signals.

\section{MR-Based Motion Correction}

We aimed to test the feasibility of using a short 1-min additional PET/MR acquisition to build a patient-specific respiratory motion model to motion-correct the previously acquired clinical PET data. To test this approach, we used an additional 4-min acquisition at the end of the scan, including MR sequences for validation and test purposes and enough dynamic MR data to build 2 motion models: 1 with 2 min $40 \mathrm{~s}$ of MR data (model ${ }_{\text {long }}$ ) and 1 with only $1 \mathrm{~min}$ of MR data $\left(\right.$ model $\left._{\text {short }}\right)$. This approach allowed us to test the performance of our motion-correction methodology with 2 hypotheses. The first hypothesis was that motion captured by the full dynamic MR sequence (model long) can successfully motion-correct simultaneously acquired PET data $\left(\mathrm{PET}_{\text {model }}\right)$, in which acquisition duration is long enough to ensure good

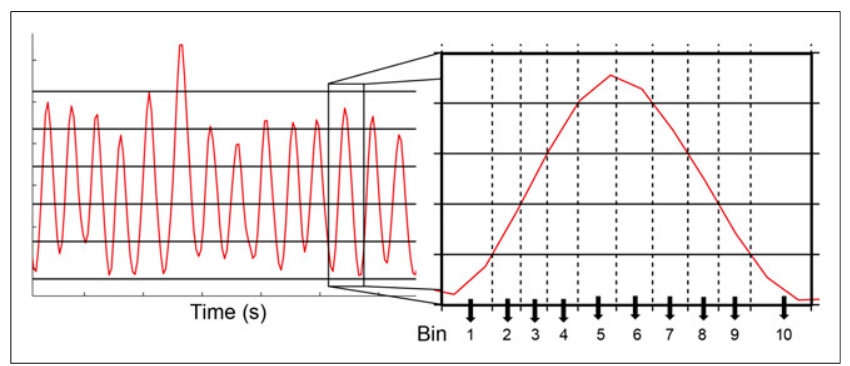

FIGURE 3. Section of respiratory signal showing amplitude-based binning and rejection scheme, with close up showing how 1 respiratory cycle is divided by amplitude, while discriminating between inhalation (positive gradient) and exhalation (negative gradient). count statistics in PET. The second hypothesis was that only $1 \mathrm{~min}$ of the MR sequence (model $\left.l_{\text {short }}\right)$ was required to capture enough motion to motion-correct PET data acquired earlier during the clinical scan $\left(\mathrm{PET}_{\text {clinical }}\right)$ (in our case with an interval of up to $1 \mathrm{~h}$ between acquisitions).

\section{Study Design: MR-Based Motion Correction}

An additional 4-min set of PET/MR sequences was acquired on a range of patients undertaking clinical PET/MR scans, covering a range of diseases and tracers $\left({ }^{18} \mathrm{~F}-\mathrm{FDG}\right.$ and ${ }^{68}$ Ga-DOTATATE) and varying length, from multibed-position whole-body scans to cardiaconly scans (Table 1). Figure 1 shows a typical clinical workflow with the additional acquired sequences, consisting of:

- PET $_{\text {model }}$ (4 min), in same bed position as previous PET $_{\text {clinical }}$ (chosen as the position affected by respiratory motion).

- MR Dixon (18 s), used by the manufacturer's software to produce an MR attenuation-correction (MRAC) $\mu$ map, acquired at endexpiration.

- MR pencil-beam navigator (30 s), later used to temporally align the PET- and MR-derived signals for compensation of differences in PET and MR system clocks.

- MR 2D multislice gradient echo ( 2 min $40 \mathrm{~s}$ ), sagittal slices at 9 slice locations, covering the thorax and abdomen (including lungs, liver, pancreas, etc.) repeated 60 times. Scan parameters: slice thickness, $10 \mathrm{~mm}$; gap between slice centers, $25 \mathrm{~mm}$; repetition time, $5.1 \mathrm{~ms}$; echo time, $2.5 \mathrm{~ms}$; flip angle, $10^{\circ}$; pixel bandwidth, $965 \mathrm{~Hz}$; matrix size, $192 \times 144$; field of view, $262 \times 349 \mathrm{~mm}$; in-plane resolution, $1.8 \times 1.8 \mathrm{~mm}^{2}$; parallel imaging acceleration factor, 3 ; acquisition time per image, $0.3 \mathrm{~s}$.

The sagittal imaging plane was chosen to minimize through slice motion. Previous studies report lung lesion displacements of only $1.2 \mathrm{~mm}$ laterally, compared with 2.2 and $5.5 \mathrm{~mm}$ in the anteriorposterior and superior-inferior directions, respectively (13).

\section{Data Processing: MR-Based Motion Correction}

Data Binning. The data workflow is shown in Figure 2. A respiratory signal was extracted from the PET $_{\text {model }}$ acquisition with PCA. An MR binning scheme was chosen to discriminate between inhalation and exhalation via gradient sign (Fig. 3). In this way, hysteresis (intracycle variation in breathing) was accommodated, which is known to be a feature of normal breathing patterns $(13,14)$. The range of signal values at which to bin the data is chosen manually for each patient dataset, discarding data that fall outside of the usual breathing of the patient. MR images were collected at 9 different slice locations and first sorted automatically according to location $l$, then into 10 respiratory bins. At each location $l$ and each respiratory bin $n$, images were averaged; then 1 image was chosen that minimized the difference between the image and the mean image, to form 1 image, $I_{l, n}$, per location and bin.

Motion Model Formation. Nonrigid 2D registration was used (minimizing a residual complexity similarity measure) (11) to find deformation fields $D_{l, n}$ between images $I_{l, n}(n \in 2 \ldots 10)$ and $I_{l, 1}$ (exhalation), chosen as the reference image, at each slice location $l$. These $2 \mathrm{D}$ deformation fields were then resampled into the 3-dimensional PET field of view, with linear interpolation used to generate vector magnitudes for between-slice voxels. This forms 1 full 3-dimensional deformation field for each bin, with a $2 \mathrm{D}$ vector at each voxel (the third vector orthogonal to the sagittal plane is set to zero). Registrations were performed to form 


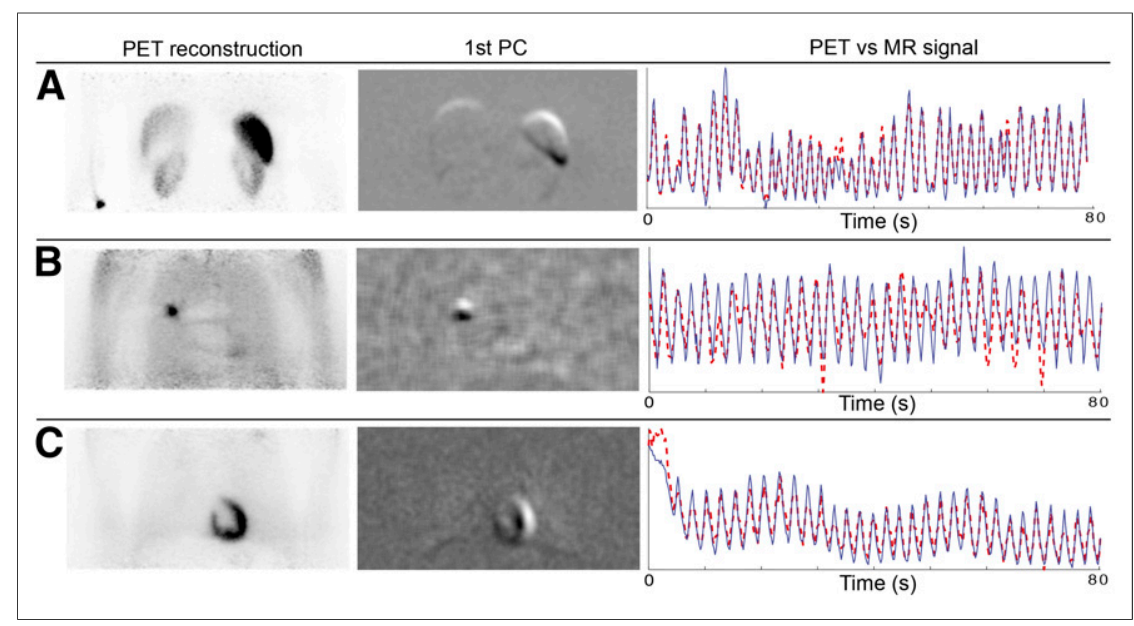

FIGURE 4. PET reconstructions (no attenuation/randoms/scatter correction), 1 view of first PC image, and 80 -s section of MR-derived (blue line) vs. PET-derived (red dotted line) respiratory signals for 3 patients with various moving anatomies. (A) Spleen. (B) Lung lesion. (C) Heart. PETderived signal is scaled to match MR-derived absolute signal for visual analysis. subset expectation maximization reconstruction algorithm was used, with 21 subsets, 5 iterations, and 4-mm gaussian postfiltering. Both PET acquisitions were reconstructed with and without motion correction. The $\mathrm{PET}_{\text {model }}$ was corrected with deformation fields from model $_{\text {long, }}$, and $\mathrm{PET}_{\text {clinical }}$ was reconstructed with deformation fields from model $_{\text {short }}$.

\section{Analysis: MR-Based \\ Motion Correction}

Motion-corrected images were compared with uncorrected images visually, with line profiles, and quantitatively with changes in SUV in a region of interest (ROI) containing areas of high tracer uptake. Measures used were maximum SUV ( $\left.\mathrm{SUV}_{\text {max }}\right)$, and peak SUV ( $\left.\mathrm{SUV}_{\text {peak }}\right)$ defined as the maximum average activity concentration within a 12-mm-diameter sphere inside the ROI (17). Focal lesions were identified and highlighted by a PET-accredited radiologist from the original clinical images of the PET/MR study. deformation fields for forward (reference to moving) motion $D_{l, n}$ and to form backward (moving to reference) motion, $D_{l, n}^{-1}$. This process was performed for both model $_{\text {short }}$ and model $_{\text {long. }}$.

Motion-Compensated PET Reconstruction. For each patient dataset, a respiratory signal was extracted from the $\mathrm{PET}_{\text {model }}$ and $\mathrm{PET}_{\text {clinical }}$ data. Both datasets were then gated using the same scheme as applied to the MR data. The gating was performed by unlisting the PET data into 10 sinograms. PET $_{\text {model }}$ data were selected as the $2 \min 40 \mathrm{~s}$ of PET that was acquired simultaneously to the motion-capturing MR data and $\mathrm{PET}_{\text {clinical }}$ as the previously acquired clinical PET data. The attenuation $\mu$ map was assigned to 1 of the gates (in the case it was not correctly acquired at end-exhale) by visual comparison with the gated MR, then 10 $\mu$ maps were formed to match the motion states of the gated PET emission data by warping with the forward-motion deformation fields. Motioncompensated image reconstruction was used to form motion-corrected PET images with randoms, attenuation, scatter processes, and motion incorporated in the system matrix of the reconstruction $(15,16)$. An ordered-

\section{RESULTS}

\section{Respiratory Signal Validation}

Over all patients, there was a strong correlation between MR pencil-beam and PET-derived signals (mean, $0.89 \pm 0.09$; range, 0.70-0.98). Figure 4 shows a reconstructed image, first PC, and section of the MR- and PET-derived signals for a range of patients.

\section{MR-Based Motion Correction}

Increases in $\mathrm{SUV}_{\text {peak }}$ and $\mathrm{SUV}_{\text {max }}$ in lesions in patients 1 and 2 are given in Table 2. First, the methodology was tested on patient 1, with just $\mathrm{PET}_{\text {model }}$ being acquired and motion correction with deformation fields from model long applied. Table 2 shows an increase in $\mathrm{SUV}_{\text {peak }}$

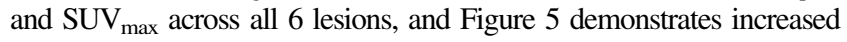
sharpness in a selection of these lesions with line profiles.

Figure 6 shows a comparison of uncorrected/corrected reconstructions in both $\mathrm{PET}_{\text {model }}$ and $\mathrm{PET}_{\text {clinical }}$ for patients 2-4, for which

TABLE 2

Increase in SUV peak $_{\text {and SUV }}$ max in Lesions in Patient 1 (Multiple Liver/Lung Lesions) and Patient 2 (Multiple Pancreatic Lesions)

\begin{tabular}{|c|c|c|c|c|c|}
\hline \multirow[b]{2}{*}{ Patient no. } & \multirow[b]{2}{*}{ Lesion no. } & \multicolumn{2}{|c|}{$\mathrm{PET}_{\text {model }}$} & \multicolumn{2}{|c|}{$\mathrm{PET}_{\text {clinical }}$} \\
\hline & & $\Delta \operatorname{SUV}_{\text {peak }}(\%)$ & $\Delta \mathrm{SUV}_{\max }(\%)$ & $\Delta$ SUV $_{\text {peak }}(\%)$ & $\Delta \mathrm{SUV}_{\max }(\%)$ \\
\hline \multirow[t]{6}{*}{1} & 1 & 10.1 & 17.6 & - & - \\
\hline & 2 & 6.2 & 2.8 & - & - \\
\hline & 3 & 11.9 & 48.0 & - & - \\
\hline & 4 & 14.6 & 24.9 & - & - \\
\hline & 5 & 7.0 & 9.3 & - & - \\
\hline & 6 & 16.9 & 22.0 & - & - \\
\hline Mean $\pm S D$ & & $11.1 \pm 4.2$ & $20.8 \pm 15.6$ & & \\
\hline \multirow[t]{4}{*}{2} & 1 & 31.9 & 42.9 & 25.0 & 49.7 \\
\hline & 2 & 9.9 & 13.9 & 8.7 & 4.5 \\
\hline & 3 & 36.9 & 57.1 & 29.8 & 36.1 \\
\hline & 4 & 13.7 & 24.0 & 6.9 & 8.4 \\
\hline Mean $\pm S D$ & & $23.1 \pm 13.3$ & $34.5 \pm 19.3$ & $17.6 \pm 11.5$ & $24.7 \pm 21.8$ \\
\hline
\end{tabular}




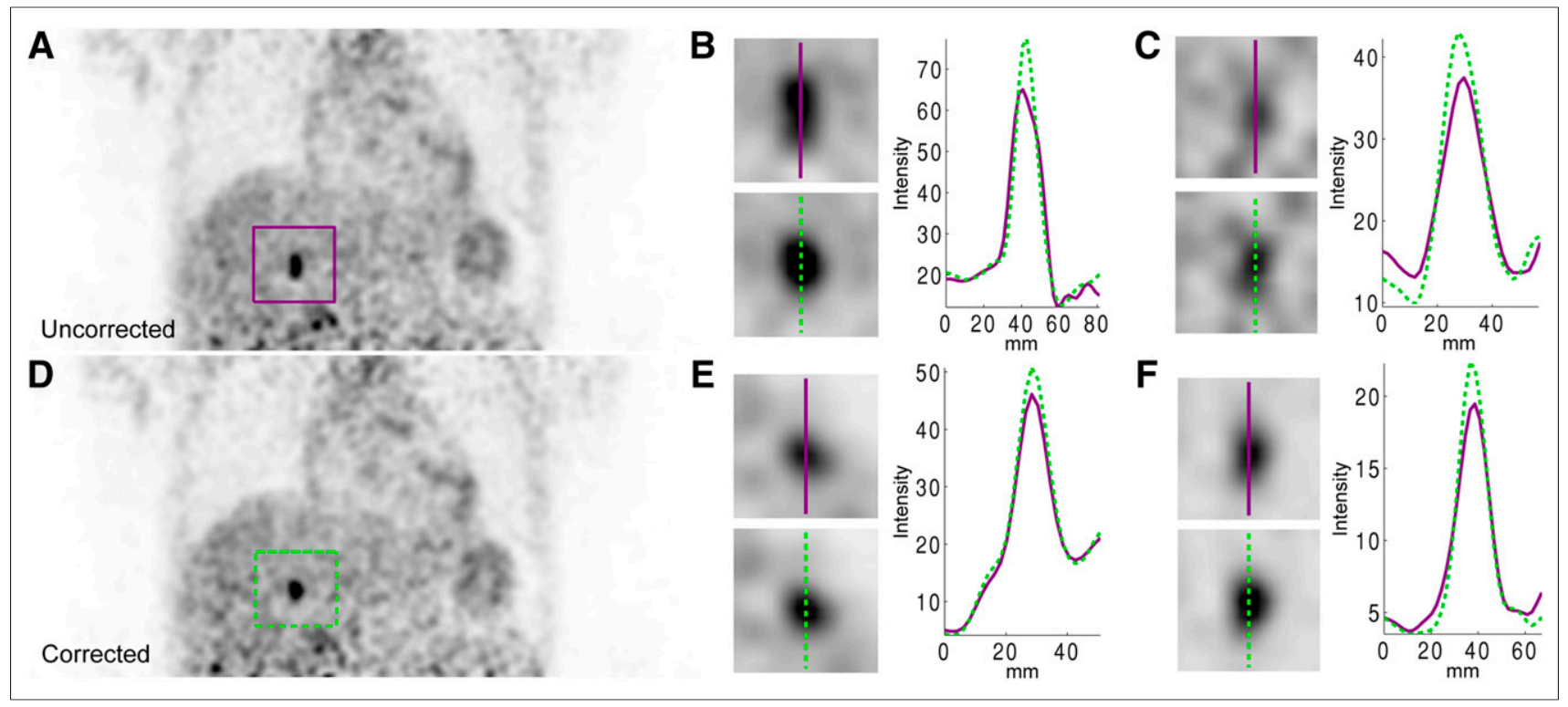

FIGURE 5. Comparison of uncorrected (A) and motion-corrected (D) reconstructions of PET model on patient 1. Each image pair shows close-up of ROI with line profiles through lesions in uncorrected image (top with purple line profile) and corrected image (bottom with dotted green line profile). Line profiles through lesions marked in $A$ and $D$ are shown in $B$, whereas line profiles through 2 other liver lesions $(C$ and $E)$ and 1 lung lesion $(F)$ are also shown.

PET $_{\text {clinical }}$ acquisitions have been corrected with motion information from 1 min worth of MR data. Figure 6A shows the uncorrected and corrected reconstruction for patient 2 in PET $_{\text {model }}$. As apparent in line profiles through 1 lesion in both $\mathrm{PET}_{\text {model }}$ and $\mathrm{PET}_{\text {clinical }}$, blurring in the original images has been reduced in the motion-corrected images.

Figure 6B shows the uncorrected/corrected reconstruction for patient 3 in the $\mathrm{PET}_{\text {model }}$. These images show an increase in sharpness and decrease in blurring of the heart. The line profiles through the heart for both $\mathrm{PET}_{\text {model }}$ and $\mathrm{PET}_{\text {clinical }}$ also show this increase in sharpness in motion-corrected images.

Figure 6C shows the uncorrected/corrected reconstructions for patient 4 in the $\mathrm{PET}_{\text {model, }}$, for which there are no avid lesions present but there is a significant motion artifact at the lungliver edge in the uncorrected image. This artifact is removed because of motion correction as the workflow allows the $\mu$ map to be assigned to the most appropriate gate. The hump marked with arrows on the uncorrected line profiles in the $\mathrm{PET}_{\text {model }}$ and $\mathrm{PET}_{\text {clinical }}$ show where the $\mu$ maps start, at points different from the actual lungliver edge.

Processing time for 1 motion-correction PET reconstruction performed offline (using nonoptimized Matlab code and STIR) was approximately $6 \mathrm{~h}$ in total.

\section{DISCUSSION}

We have demonstrated increased sharpness and quantitative change in PET images with a practical motion-correction scheme in several patients in vivo. These have the potential to improve lesion delineation and quantitation accuracy and may also contribute to improved lesion detectability. Our methodology also demonstrated respiratory artifact reduction, such as evident in patient 4 at the lung-liver edge, for which the common banana artifact is observed. This artifact can occur where there is a mismatch in respiratory position between MRAC acquisition and emission data when the MRAC is acquired at inhale position, rather than exhale as requested by the radiographer.

The 3 examples of $\mathrm{PET}_{\text {clinical }}$ for patients $2-4$ were acquired between 50 and $61 \mathrm{~min}$ before the motion model sequence acqui- sition. For each patient-specific motion model to be applicable an hour before formation, it is assumed that the patient is in the same position in the scanner and breathing style is consistent. Furthermore, we collect only a 1-dimensional respiratory signal, which does not discriminate between types of breathing. Other methods have been proposed in the literature that collect data during different types of breathing to account for intercycle variability (9), but this increases scan time and can cause extra patient discomfort by forcing different types of breathing. The current methodology also does not account for bulk motion during the scan; nevertheless, we demonstrated a clear improvement in the corrected PET $_{\text {clinical }}$ images, suggesting that even when the motion model data are acquired up to an hour later, image quality can be improved. With the current methodology, if the amplitude of respiration is larger in the $\mathrm{PET}_{\text {clinical }}$ than $\mathrm{PET}_{\text {model }}$, then data that fall outside of the predetermined amplitude range are currently rejected, leading to a small loss of count statistics.

The extra 1-min PET/MR motion model sequence could be acquired immediately after the routine clinical protocol (as per the methodology in this work) or could be incorporated within the clinical protocol. The duration of PET to be motion-corrected can range from $3 \mathrm{~min}$ per bed position for whole-body scans to up to 60-90 min for research studies with 1 bed position. For a whole-body scan with 4 bed positions, 2 of the positions adversely affected by motion (thorax and abdomen) may be extended by 1 min each (Fig. 7). The PET part of the motion model acquisition can therefore not only be used to provide a respiratory signal but also be included with the other 3 min of PET data, ensuring greater count statistics. In some clinical protocols, the duration of MR acquisition may be shorter than the PET duration, in which case the motion-capturing MR sequence can be acquired with no time penalty. Acquiring the extra MR close in time to the clinical data reduces the chance of a change in breathing pattern or patient motion, reducing the validity of the motion model.

Currently MR data are collected at 9 sagittal slice locations but data at the outer locations are often redundant as they image only nonmoving sections at the edges of the body. Using fewer slice locations could result in a more efficient acquisition, reducing the 


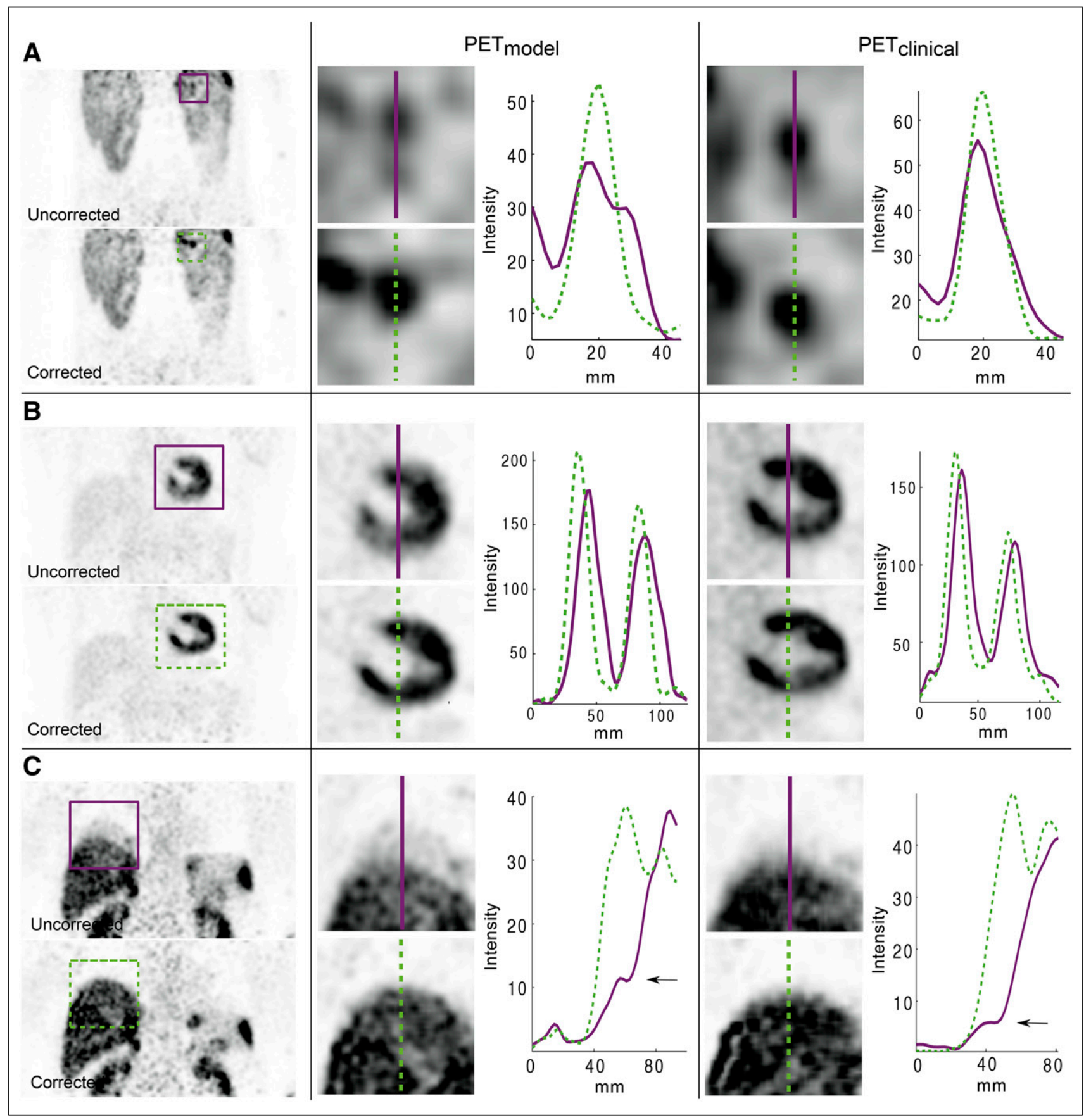

FIGURE 6. Comparison of uncorrected and motion-corrected reconstructions of $\mathrm{PET}_{\text {model }}$, with line profiles through ROls for $\mathrm{PET}$ model and $\mathrm{PET}_{\text {clinical }}$ on patient 2 (A), patient $3(\mathrm{~B})$, and patient 4 (C). For each image pair, uncorrected images are top with purple line profiles, and corrected images are bottom with dotted green line profiles.

additional scan time further to under $1 \mathrm{~min}$. As the gradient echo MR sequence covers a greater field of view in the superior-inferior direction than the PET field of view, motion information from this acquisition could be applied to multiple PET bed positions, enhancing efficiency further. However, as the PET-derived respiratory signals from different bed positions would be derived from different moving anatomies, this concept would need to be investigated further.

We have examined only SUV changes in lesions that are already avid and detected in the uncorrected images by the radiologist. Detectability becomes a more important issue for smaller lesions that go undetected in uncorrected images but have the potential to become visible with motion correction. Quantitative improvements in lesion detectability have been shown with MR-based PET motion correction in simulated thoracic lesions (15) and in hepatic lesions in rabbit and primate studies (7). The impact on detectability within a clinical environment requires further study.

\section{CONCLUSION}

We have demonstrated that a respiratory signal can be obtained from raw PET data, comparable with a gold-standard MR pencil-beam 


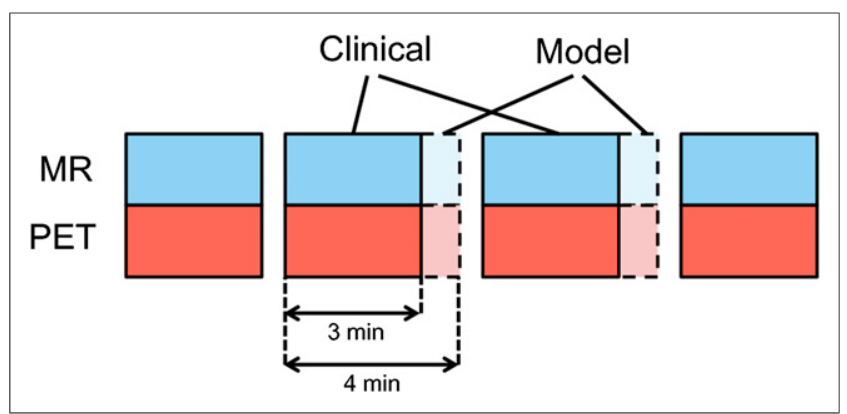

FIGURE 7. Proposed whole-body clinical workflow with extended PET bed position durations for motion model acquisitions.

navigator. We have then proposed a practical, anatomy-independent MR-based correction strategy for PET data affected by respiratory motion and have shown it can improve image quality for PET acquired simultaneously to the motion-capturing MR and furthermore for PET acquired earlier during a clinical scan while any other free-breathing or breath-hold diagnostic MR is being acquired. Our method does not require external hardware or any change to the clinical protocol, except for an extra short acquisition at the end of a clinical protocol. The method will have potential benefit for a wide range of oncologic and cardiac applications of PET/MR.

\section{DISCLOSURE}

The costs of publication of this article were defrayed in part by the payment of page charges. Therefore, and solely to indicate this fact, this article is hereby marked "advertisement" in accordance with 18 USC section 1734. Support for this study is from Siemens/UCL IMPACT studentship, the EPSRC (EP/K005278/1), and support by The National Institute for Health Research University College London Hospitals Biomedical Research Centre. No other potential conflict of interest relevant to this article was reported.

\section{REFERENCES}

1. Nehmeh SA, Erdi YE. Respiratory motion in positron emission tomography/ computed tomography: a review. Semin Nucl Med. 2008;38:167-176.

2. Bai W, Brady M. Motion correction and attenuation correction for respiratory gated PET images. IEEE Trans Med Imaging. 2011;30:351-365.

3. Nehmeh SA, Erdi Y, Ling CC, et al. Effect of respiratory gating on quantifying PET images of lung cancer. J Nucl Med. 2002;43:876-881.

4. Boucher L, Rodrigue S, Lecomte R, Bénard F. Respiratory gating for 3-dimensional PET of the thorax: feasibility and initial results. J Nucl Med. 2004;45:214-219.

5. Klein G, Reutter B, Huesman R. Non-rigid summing of gated PET via optical flow. IEEE Nucl Sci Symp Conf Rec. 1996;1339-1342.

6. Guérin B, Cho S, Chun SY, et al. Nonrigid PET motion compensation in the lower abdomen using simultaneous tagged-MRI and PET imaging. Med Phys. 2011;38:3025-3038.

7. Chun SY, Reese TG, Ouyang J, et al. MRI-based non-rigid motion correction in simultaneous PET/MRI. J Nucl Med. 2012;53:1284-1291.

8. Würslin C, Schmidt H, Martirosian P, et al. Respiratory motion correction in oncologic PET using T1-weighted MR imaging on a simultaneous whole-body PET/MR system. $J$ Nucl Med. 2013;54:464-471.

9. King AP, Buerger C, Tsoumpas C, Marsden PK, Schaeffter T. Thoracic respiratory motion estimation from MRI using a statistical model and a 2-D image navigator. Med Image Anal. 2012;16:252-264.

10. Thielemans K, Tsoumpas C, Mustafovic S, et al. STIR: software for tomographic image reconstruction release 2. Phys Med Biol. 2012;57:867-883.

11. Myronenko A, Song X. Intensity-based image registration by minimizing residual complexity. IEEE Trans Med Imaging. 2010;29:1882-1891.

12. Thielemans K, Rathore S, Engbrant F, Razifar P. Device-less gating for PET/CT using PCA. IEEE Nucl Sci Symp Conf Rec. 2011;3904-3910.

13. Seppenwoolde Y, Shirato H, Kitamura K, et al. Precise and real-time measurement of 3D tumor motion in lung due to breathing and heartbeat, measured during radiotherapy. Int J Radiat Oncol Biol Phys. 2002;53:822-834.

14. McClelland JR, Hawkes DJ, Schaeffter T, et al. Respiratory motion models: a review. Med Image Anal. 2013;17:19-42.

15. Polycarpou I, Tsoumpas C, Marsden PK. Analysis and comparison of two methods for motion correction in PET imaging. Med Phys. 2012;39:64746483.

16. Tsoumpas C, Polycarpou I, Thielemans K, et al. The effect of regularization in motion compensated PET image reconstruction: a realistic numerical 4D simulation study. Phys Med Biol. 2013;58:1759-1773.

17. Boellaard R, Delgado-Bolton R, Oyen WJG, et al. FDG PET/CT: EANM procedure guidelines for tumour imaging: version 2.0. Eur J Nucl Med Mol Imaging. $2015 ; 42: 328-354$ 